\title{
LEI GERAL DE PROTEÇÃO DE DADOS (LGPD): O DESAFIO DE SUA IMPLANTAÇÃO PARAA SAÚDE
}

\section{GENERAL DATA PROTECTION REGULATION (GDPR): THE CHALLENGE OF ITS IMPLEMENTATION FOR HEALTH}

\author{
Larissa Oliveira Hawryliszyn ${ }^{1}$ \\ Natalia Gavioli Souza Campos Coelho ${ }^{2}$ \\ Paulo Roxo Barja $^{3}$
}

Resumo: Em 2020, entrou em vigor a Lei Geral de Proteção de Dados (LGPD), que havia sido aprovada em 2018, com a finalidade de promover a proteção dos dados pessoais de todos os cidadãos que estejam no Brasil, afetando diferentes setores e serviços. No presente trabalho, buscamos discutir a aplicabilidade da LGPD no setor da saúde, uma vez que este pode ser considerado o setor de adequação mais complexa a esta legislação, por trabalhar a todo momento com dados sensíveis. Durante o estudo, foi possível detectar situações em que, ao mesmo tempo, as informações do paciente auxiliam em seu cuidado, mas geram dificuldades para adequação da lei. Esta implementação da LGPD requer cuidado e envolvimento de toda a instituição, o cumprimento de boas práticas, estruturadas e mantidas por uma governança que se preocupa com normas de segurança, padrões técnicos, ações educativas, fiscalizações internas, assim como mapeamento e ações de mitigação de riscos.

Palavras-chave: LGPD. Saúde. Processo. Proteção. Cultura.

Abstract: In 2020, the General Data Protection Regulation (GDPR) came into force, which had been approved in 2018, with the purpose of promoting the protection of the personal data of all citizens in Brazil, affecting different sectors and services. In the present work, we aim at discussing the applicability of GDPR in the health sector, since this can be considered the sector with the most complex adaptation to this legislation, for working with sensitive data every moment. During the study, it was possible to detect situations in which, at the same time, the patient's information helps in their care, but it brings difficulties to conduct the adaptation to the law. The implementation of GDPR requires care and participation of the entire institution, compliance with good practices, structured and maintained by a governance that is concerned with safety standards, technical standards, educational actions, internal inspections, as well as mapping and risk mitigation actions.

Key words: LGPD. Health. Process. Protection. Culture.

Data de submissão: 19.02 .2021

Data de aprovação: 30.08.2021

\footnotetext{
${ }^{1}$ Discente da Especialização em Administração Hospitalar/UNIVAP, E-mail: larissa.hawryliszyn@gmail.com.

${ }^{2}$ Discente da Especialização em Administração Hospitalar/UNIVAP, E-mail: na gavioli@hotmail.com

3 Docente-pesquisador da FEAU/Univap, E-mail: barja@univap.br.
} 
Identificação e disponibilidade:

(https://revista.univap.br/index.php/revistaunivap/article/view/2589, http://dx.doi.org/10.18066/revistaunivap.v27i54.2589).

\section{INTRODUÇÃO}

A privacidade é algo que sempre foi buscado por todas as pessoas. Em 15 de Dezembro de 1890, foi publicado por Warren e Brandeis um artigo na Harvard Law Review (EUA), intitulado O Direito à Privacidade (The Right To Privacy). Warren e Brandeis escreveram que a privacidade é o "direito de ser deixado em paz" e é focada na proteção dos direitos das pessoas (WARREN; BRANDEIS, 1890). De fato, historicamente o direito à privacidade está conectado, interligado com as tecnologias da informação, que permitem grandes benefícios para a saúde e devem trazer segurança aos dados pessoais (SARLET; MOLINARO, 2019).

A partir de 1992, começa a ocorrer o (rápido) processo de popularização da internet. Com o lançamento da WWW (World Wide Web), usuários ao redor do mundo passaram a poder obter (muitas vezes em tempo real) informações antes inacessíveis, o que possibilitou um compartilhamento e fluxo contínuo de trocas de informações (inclusive pessoais) através da rede.

Em novembro de 2010, teve início no Brasil o debate sobre a proteção de dados pessoais, com o propósito de se elaborar uma lei específica sobre o tema (JINKINGS, 2010). Em 18 de novembro de 2011, foi sancionada a Lei de Acesso à Informação (LAI) de $\mathrm{N}^{0} .12 .527$, que dispõe sobre dados pessoais de acesso público (BRASIL, 2011). Em 2012, foi sancionada a Lei Carolina Dieckmann de Nº.12.737 que é a tipificação de crimes cibernéticos, como compartilhar dados sem autorização (BRASIL, 2012). Em 2014, entrou em vigor o Marco Civil da Internet que é a lei de №.12.965, que regula o uso da Internet no Brasil por meio da previsão de princípios, garantias, direitos e deveres para quem usa a rede, bem como da determinação de diretrizes para a atuação do Estado (BRASIL, 2014). Em 2016, foi aprovado o Regulamento geral sobre a proteção de dados (GPDR) de número 2016/679, que entrou em vigor em maio de 2018 (UNIÃO EUROPEIA, 2018).

Escândalos como o do Facebook-Cambridge, de uso ilícito de dados de usuários da rede social (LUIZ; RODRIGUES, 2018), assim como o vazamento de dados de pacientes com cadastro no Cartão Nacional de Saúde (DADOS, 2017), chamaram a atenção das autoridades brasileiras, acelerando as votações no Senado para a Lei Geral de Proteção de Dados (LGPD), que foi sancionada em agosto de 2018 após unificação dos textos da Câmara e do Senado no PLC n 53 (INFORMATIVO, 2018). Além de estabelecer normas para garantir a privacidade e o uso de dados pessoais na internet, a nova lei também impulsionou a criação de toda uma infraestrutura de segurança. Uma prova disso é a ANDP (Autoridade Nacional de Proteção de Dados), cuja função é garantir a observância à LGPD (NASCIMENTO, 2018).

O presente artigo identifica os principais desafios em potencial para a adequada aplicação da lei no setor da Saúde e propõe estratégias para sua implementação, com o objetivo de auxiliar as instituições hospitalares no cumprimento efetivo das exigências da LGPD em sua totalidade. 


\section{METODOLOGIA}

Foi realizada pesquisa bibliográfica combinando os termos "LGPD", "Lei Geral de Proteção de Dados", "LGPD na Saúde" e "Impactos LGPD na Saúde", dois a dois. As pesquisas foram realizadas em diferentes fontes: Google Acadêmico, Scielo, revistas científicas da área da Saúde e indexadas no sistema Qualis Capes (Revista de Saúde Pública, Revista Brasileira de Ciência e Saúde, Revista de Administração Hospitalar, Revista da Fiocruz, Inovação em Saúde, Ciência e Saúde). Adotou-se, como critérios de inclusão: i) o período de publicação (os artigos precisariam ter sido publicados no período entre 2018 e 2020); e ii) a relevância do artigo quanto ao tema, considerando-se mais relevantes os artigos que mencionassem o tema no título e tratassem explicitamente da necessidade de adequação das instituições de saúde para implementação da lei. Posteriormente, buscou-se materiais complementares, publicados na internet também no período 2018-2020, para complementar a discussão.

Os autores do presente artigo participaram de workshops de adequação da LGPD e, também, de seminários promovidos por empresas da área da saúde, buscando embasamento para aprofundar a análise da aplicabilidade da lei. Semanalmente, a equipe mantinha contato com o coordenador do trabalho, através do aplicativo Zoom.

Foi realizada a análise crítica da LGPD à luz da pesquisa bibliográfica efetuada, com especial atenção dada aos termos jurídicos; obrigatoriedades das empresas, sistemas de verificações e sanções para o não cumprimento das normas foram os principais tópicos verificados. Com base na análise da lei, promoveu-se o debate entre os pesquisadores e o gestor de tecnologia da informação de uma rede hospitalar no município de São José dos Campos, responsável pela implementação da Lei na instituição, com quem discutimos os processos a serem levantados e implementados para o cumprimento da lei, bem como seus potenciais impactos na área da saúde. O debate ocorreu em junho de 2020 e foi efetuado na forma remota/digital.

Por fim, foi realizada entrevista com o gestor de tecnologia da informação da instituição hospitalar filantrópica do município citado à qual parte dos autores são vinculados profissionalmente, para entender como a LGPD afeta a instituição e como esta tem se preparado para cumprir as novas demandas legais.

\section{RESULTADOS E DISCUSSÃO}

\subsection{COLETA/SELEÇÃO DE MATERIAL}

Nas pesquisas bibliográficas efetuadas, verificou-se que a maior parte das revistas acadêmicas consultadas não havia publicado artigos sobre o tema no intervalo cronológico considerado. Uma exceção foi a Revista Fiocruz, que permitiu encontrar quatro registros utilizando o termo LGPD, sendo três registros de 2018 e um de 2019. Destaca-se entre eles o artigo intitulado "Marcos legais nacionais em face da abertura de dados para pesquisa em saúde: dados pessoais, sensíveis ou sigilosos e propriedade intelectual", publicado em 2018 e selecionado para análise. No Google Acadêmico, encontrou-se um total de 960 resultados; após filtragem por período cronológico (2018-2020) e relevância, foram selecionados para análise três artigos: 
i) "Os impactos da Lei Geral de Proteção de Dados Pessoais na Saúde Suplementar" (GREGORI, 2020);

ii) "Questões Tecnológicas, Éticas e Normativas da Proteção de Dados Pessoais na Área da Saúde em um Contexto de Big Data" (Revista Brasileira de Direitos Fundamentais e Justiça, 2019);

ii) "A era biotecnológica: apontamento sobre os dados genéticos na LGPD (Lei Geral de Proteção de Dados)", publicado no XIV EVINCI (Evento de Iniciação Científica, UniBrasil, Curitiba, 2019).

Pesquisando com o termo de busca "LGPD" no site da Scielo Saúde Pública, encontramos um artigo publicado em dezembro de 2019, "A lei geral de proteção de dados pessoais em empresas brasileiras: uma análise de múltiplos casos" (PIURCOSKY et al, 2019). Já utilizando o termo "Lei Geral de Proteção de Dados, o site traz quatro artigos, incluindo um do Chile e um de Portugal.

Adicionalmente, as pesquisas permitiram obter acesso também a diversas cartilhas com práticas de boa convivência; dentre elas, destacamos: i) a da ANAHP (Associação Nacional de Hospitais Privados), que demonstra uma visão da lei no do ponto de vista da gestão hospitalar, destacando oito estratégias como fator de redução de possíveis multas; e ii) a da Fundação Vanzolini, que traz um guia de implantação escrito num grau de detalhe suficiente para a aplicação da Lei na instituição.

\subsection{O QUE DIZEM OS ARTIGOS}

Segundo Gregori (2020), a Constituição de 1988 foi a primeira lei no Brasil a preservar a dignidade da pessoa humana, garantindo a liberdade de expressão e o direito de ir e vir. Com o passar dos anos, o mundo foi evoluindo e com essa evolução houve a necessidade de serem criadas novas leis que protejam o cidadão; um exemplo disso foi o código de defesa do consumidor (GREGORI, 2020). De acordo com Gregori, o setor jurídico brasileiro deve estar sempre atento aos avanços tecnológicos que alteram (muitas vezes de modo positivo) a vida dos cidadãos no cotidiano, a forma de trabalho, a economia - mas que, por outro lado, podem aumentar a exposição de seus dados pessoais.

O avanço tecnológico conectou o mundo de forma ágil (ainda que nem sempre responsável); a tecnologia também trouxe ferramentas que permitem melhorias qualitativas de gerenciamento, como o uso de algoritmos que racionaliza, padroniza e melhora a qualidade de muitos processos de trabalho (SARLET; MOLINARO, 2019). Esse grande aumento tecnológico de conectividade e circulação de informação, no entanto, trouxe preocupação aos juristas, pois as informações genéticas possuem relevância para empresas de planos de saúde, seguro de vida e mesmo as indústrias farmacêuticas. O cruzamento de dados, que se faz possível através da inteligência artificial, potencializa a exposição de dados que podem violar a integridade física e moral dos indivíduos. Um dos fundamentos da LGPD é garantir que os dados pessoais e sensíveis não sejam comercializados entre empresas e sim utilizados apenas para os fins coletados, garantindo a segurança física e moral dos donos dos dados (SCHRAMM, 2020).

$\mathrm{Na}$ saúde suplementar, tanto operadoras de planos de saúde como prestadores de serviços e estipulantes de planos coletivos são os responsáveis pelas informações que dispõem de terceiros e pelas decisões referentes ao tratamento de dados pessoais, assim como as instituições hospitalares (GREGORI, 
2020). Todas as regras que garantem a segurança do paciente e que já estavam em vigência neste setor antes da aprovação da LGPD continuarão a ser aplicadas, sendo agora complementadas pela nova legislação.

Em artigo publicado há alguns anos (SOUZA et al., 2018), equipe da Fiocruz já se referia à lei como um novo pacto capaz de definir as bases do tratamento de dados pessoais, procurando garantir tanto a preservação dos direitos e liberdades dos cidadãos quanto proporcionar segurança para os que realizam o tratamento de dados de forma legítima e transparente. A LGPD estabelece normas específicas para o tratamento de informações pessoais para pesquisa pública em saúde, proporcionando o espaço necessário para que o desenvolvimento de reflexão crítica e de normativa específica referente à matéria seja empreendido em um futuro imediato (SOUZA et al., 2018).

\subsection{O QUE DIZEM AS CARTILHAS}

É crescente a utilização dos recursos da Tecnologia da Informação e Comunicação na área da Saúde, onde dados transitam em grande volume (e nem sempre de forma ordenada), sendo usados em recursos como prontuário eletrônico do paciente (PEP), telemedicina, troca de informações entre instituições e na área assistencial, entre outras. Com isso, é real a necessidade de padronização e regulamentação para a correta utilização de tais dados (ANAHP, 2019).

Os princípios da segurança da informação têm por objetivo proteger os dados contra acessos não autorizados e manter a disponibilidade para os donos dos dados. O bem mais precioso das empresas são os seus bancos de dados, o que faz com que estas apliquem práticas de segurança de informação para reduzir os riscos associados ao tráfego de informação em formato digital. A tecnologia da informação está associada a uma das melhores maneiras de formular políticas para efetivar a proteção de dados dos clientes e garantir o correto manuseio (PIURCOSKY et al, 2019).

Segundo a ANAHP, a LGPD é benéfica para a sociedade, pois estabelece processos, papéis e dá transparência às relações. A cartilha da ANAHP traz oito estratégias para a implementação da lei no âmbito hospitalar, com intuito de minimizar multas aplicadas pela ANDP. O Quadro 1, a seguir, apresenta as oito estratégias. 
Quadro 1 - Estratégias de implantação da LGPD.

\begin{tabular}{|c|l|}
\hline ESTRATÉGIAS & \multicolumn{1}{|c|}{ Descrição } \\
\hline Conscientização & Conscientizar toda a equipe do que é a lei e da sua importância \\
\hline Criação de políticas & Criar políticas de segurança de dados \\
\hline $\begin{array}{c}\text { Levantamento das } \\
\text { interfaces de troca de } \\
\text { informação }\end{array}$ & $\begin{array}{l}\text { Os controles de proteção devem estar baseados no mapeamento } \\
\text { dos processos de armazenamento, processamento e transferência } \\
\text { de dados. }\end{array}$ \\
\hline $\begin{array}{c}\text { Monitoração e proteção } \\
\text { contínua }\end{array}$ & $\begin{array}{l}\text { Garantir a segurança da informação por meio de monitoramento 24h } \\
\text { por dia }\end{array}$ \\
\hline $\begin{array}{c}\text { Gestão de Consentimento } \\
\text { Criptografias }\end{array}$ & $\begin{array}{l}\text { Interface para que o indivíduo possa autorizar, bloquear ou revogar o } \\
\text { consentimento para o tratamento de dados pessoais a qualquer } \\
\text { momento }\end{array}$ \\
\hline Desenvolvimento seguro & $\begin{array}{l}\text { A criptografia garante a proteção dos dados para que estes não } \\
\text { consigam ser decodificados em caso de vazamento }\end{array}$ \\
\hline $\begin{array}{c}\text { Ações de revisão e adequações. PDCA, do inglês Plan - Do - } \\
\text { Check - Act (Planejar, fazer, verificar, agir). O PDCA é um método } \\
\text { interativo de gestão, utilizado para controle e melhoria. }\end{array}$ \\
\hline Garantia & $\begin{array}{l}\text { A segurança dos dados deve ser garantida, até mesmo em uma } \\
\text { possível queda de sistema, o dono dos dados estejam protegidos. }\end{array}$ \\
\hline
\end{tabular}

Fonte: Adaptado pelos autores (ANAHP, 2019).

A LGPD tem como objetivo proteger os dados pessoais de pessoas físicas e se apresenta dividida em vários capítulos. O guia de implantação da LGPD (GARCIA et al, 2020) permite um entendimento detalhado destes capítulos.

O capítulo I trata sobre as disposições gerais da lei, definindo a natureza dos conceitos e a importância de sua aplicabilidade. Identifica-se como Titular o paciente, Controlador o hospital, Operador os colaboradores e/ou terceiros, Encarregado de Dados o responsável indicado pelo hospital como canal de comunicação entre hospital, paciente ANPD (Autoridade Nacional de Proteção de Dados) que é órgão público que fiscaliza o cumprindo desta lei. Observa-se que todos os profissionais precisam estar envolvidos e engajados nessa adequação. Desde a entrada do paciente na recepção até a sua alta, quando seu prontuário é encaminhado para fins administrativos, estão sendo trabalhados dados sensíveis.

O capítulo II dedica-se ao consentimento do paciente (ou responsável legal), necessário para que o hospital colete os dados, efetue o tratamento e utilize estes dados para cuidar do paciente. É importante que o hospital informe a necessidade da obtenção destes dados e de que forma serão utilizados durante seu quadro clínico, uma vez que o paciente pode exigir a exclusão de suas informações no prontuário a qualquer momento. É preciso demonstrar que todas as informações disponibilizadas no prontuário estão protegidas.

O capítulo III informa os direitos dos titulares e explicita a exigência de que Controlador e Operador tenham uma gestão rigorosa quanto ao modo como os dados são tratados. Entende-se que o paciente pode confirmar o consentimento da obtenção de seus dados, revogar esse consentimento, solicitar correção, anonimização, bloqueio ou eliminação. O capítulo IV refere-se ao tratamento dos dados pelo Poder Público e o capítulo $\mathrm{V}$ trata da transferência internacional dos 
dados.

O capítulo VI descreve deveres e responsabilidades do Controlador, Operador e Encarregado. Este último é uma nova função que a lei solicita às empresas, e que pode ser um colaborador próprio ou terceiros. Em hospital, por ser um ambiente com processos complexos, é interessante ser um colaborador próprio, que entenda a empresa e saiba articular as necessidades dos pacientes, sem deixar impactar os processos hospitalares. Um terceiro iria cumprir as solicitações e exigências necessárias, mas provavelmente não teria a visão global da instituição.

O capítulo VII aborda a Segurança e Boas Práticas, informando as medidas de segurança e padrões que as empresas precisam realizar para proteger os dados. A ANPD irá monitorar esses tratamentos; assim, é importante que todas as instituições se adequem a estes padrões e realizem ações periódicas de monitoramento do tratamento de dados, para que possam responder pelas informações armazenadas.

O capítulo VIII fala da fiscalização quanto ao cumprimento da lei, ressaltando as sanções administrativas que poderão ser aplicadas pela ANPD. O capítulo IX complementa o anterior, determinando as responsabilidades da ANPD e do Conselho Nacional de Proteção de Dados Pessoais e da Privacidade (CNPDPP). Pensando em hospitais, que geralmente precisam buscar melhores processos para obter maior receita, é de extrema importância a adequação à lei, para garantir processos mais seguros e informações fidedignas, evitando possíveis penalizações financeiras.

Por fim, através de seu guia de implantação, a Fundação Vanzolini informa que, para uma organização atender continuamente e de maneira sustentável aos requisitos da LGPD, será preciso implementar um sistema de gestão (constituído por processos, pessoas e tecnologias) que permeie todas as áreas de negócio.

\subsection{ENTREVISTA}

Em entrevista presencial previamente autorizada, o responsável pela implantação da LGPD na instituição filantrópica, à qual são vinculados autores deste estudo, foi questionado sobre como os hospitais estão se preparando após o Senado ter retirado o adiamento da vigência para maio de 2021. Segundo o entrevistado, a instituição dividiu a implantação em duas etapas. A primeira etapa está sendo chamada de escudo, com o propósito de blindar a instituição nos primeiros 60 dias desde a entrada em vigor da lei (em agosto), elencando as prioridades de maior impacto e atuando nelas imediatamente. A segunda etapa vem sendo chamada de jornada LGPD e se volta à implantação de todas as demais alterações que serão necessárias. Quando questionado a respeito de qual seria o maior desafio quanto à adequação a esta lei na área da saúde, sua resposta foi, de imediato, "conscientização das pessoas".

A LGPD é um tripé: tecnologia, processos e pessoas. Temos que ter a tecnologia protegendo, os processos sendo ajustados para proteger os dados dos titulares, mas sem as pessoas, pode ser a empresa com o maior dinheiro, com a maior tecnologia, com o maior investimento, se as pessoas não estiverem comprometidas você não protege dado pessoal de ninguém. (Gestor TI, comunicação pessoal, 2020)

Levando em consideração a estratégia de escudo citada pelo gestor, questionamos os primeiros impactos que percebeu durante esta implantação: 
O impacto maior é que, para realizar a adequação, precisa das pessoas que estão nas pontas; era necessário que a informação chegasse às pontas, que todos soubessem o que é a LGPD. Foi realizado workshop nas casas e formado um comitê de LGPD para cada casa. O comitê será o responsável por replicar a informação do que é a lei e quais os impactos ela traz para o trabalho de cada um. (Gestor TI, comunicação pessoal, 2020)

O entrevistado também explicou como a lei atuará nos hospitais e como a ANAP fará as auditorias, que serão baseadas nos relatórios de impacto:

\begin{abstract}
[A lei] Vai punir quem não fizer o trabalho de casa. Em um webinar que eu assisti, uma advogada especializada em LGPD deu um exemplo de dois hospitais, um hospital rico, [...] informação 100\% segura, tudo digital, focaram na segurança dos seus sistemas e outro hospital pobre, sem dinheiro, tudo no papel. Os dois entraram na LGPD, o hospital rico não se preocupou e não foi atrás dos seus GAPS ${ }^{4}$. O hospital pobre correu atrás [...] documentou tudo, se planejando para investir. Vazou informação dos dois hospitais, a ANPD chegou para fazer a auditoria e não multou o hospital pobre, pois ele tinha todas as vulnerabilidades mapeadas com planejamentos de melhorias, já o hospital rico, por achar que seus sistemas estavam seguros, não tinha nenhuma vulnerabilidade mapeada. (Gestor TI, comunicação pessoal, 2020)
\end{abstract}

Questionado sobre como deverá ocorrer a segunda etapa, chamada de Jornada LGPD, o entrevistado explicou que, após a conscientização das pessoas a respeito da lei e de seus impactos, virá o mapeamento de dados, onde serão identificadas as vulnerabilidades, a mão de obra e os investimentos necessários para adequação. A estratégia de escudo trará um mapeamento dos processos mais vulneráveis e de escala maior.

Em seu artigo 41, a lei exige que as empresas tenham um DPO (Data Protection Officer), que é uma figura (de natureza jurídica ou não) responsável pela proteção dos dados. Apesar dessa função/cargo ser muito recente, várias empresas estão aproveitando a oportunidade e oferecendo o serviço de DPO. O DPO é a referência da política de privacidade de dados da instituição, a terceirização tem suas vantagens e desvantagens e todos os pontos devem ser analisados, pois existe a responsabilidade de conhecer toda a cultura da empresa, todo o perfil dos funcionários e a confiança. Além disso, a figura do DPO tem a responsabilidade de gerenciar todos os dados e o eventos, apresentando relatórios, medidas preventivas e corretivas. Para realizar toda a gestão, já existem sistemas que auxiliam nos controles, pois em uma instituição hospitalar, a quantidade de dados sensíveis torna humanamente impossível gerenciar tudo manualmente (NASCIMENTO, 2018).

A partir de todos os pontos abordados, como identificar que a LGPD está implantada e como avaliar sua eficácia? Responde o entrevistado:

A LGPD vai estar implantada quando chegar na fase de monitoramento. Tem todo um trabalho de preparação, conscientização, depois analisar onde estão nossos gargalos, seja ele físico, tecnológico ou mesmo de pessoas, fazer um mapeamento dos dados, revisar e aditar os contratos, criar uma política de privacidade, política de cookies nos sites, $\mathrm{N}$ tarefas a serem cumpridas e quando todas forem cumpridas a gente entra na fase de monitoramento, como se fossem rodas, um PDCA. Da LGPD nasce um projeto e quando ela acaba vira um programa (...) A partir do ano que vem, vamos ter

\footnotetext{
${ }^{4}$ GAPs: Gap é originária de uma palavra inglesa a qual possui como principal significado vão, brecha ou até mesmo lacuna. Esta palavra também é muito utilizada como um significado de diferença.
} 
departamentos de LGPD, assim como (o departamento de) qualidade surgiu nos hospitais há 20, 30 anos. (Gestor TI, comunicação pessoal, 2020)

Em relação ao orçamento para implementação da lei, como a lei é muito recente, as instituições em geral não possuem um orçamento definido para esta implementação. Foram realizadas algumas cotações de consultorias para melhor compreensão do mercado. O mapeamento de investimento está sendo realizado e planejado.

Perguntamos também ao entrevistado sobre sua opinião a respeito da lei trazer benefícios ou malefícios para o setor:

[A lei] vai trazer benefícios. Eu falo que leis importantes surgem depois de
um ou mais acontecimentos que prejudicaram empresas, pessoas ou
nações, você pega, por exemplo, a lei sobre os direitos humanos que surgiu
após a segunda guerra mundial [...]. A lei será benéfica para dar segurança,
os princípios da lei foram muito bem pensados então não tenho dúvida que
trará benefícios. A sua adequação, quando encarada como uma mera
obrigatoriedade para obedecer uma lei, desvia sua função, pois não vai dar
a real importância da privacidade dos dados [...]. (Gestor TI, comunicação
pessoal, 2020)

Dentro dos hospitais existe a checagem de paciente, onde muitas vezes é dito em voz alta o nome completo do paciente, para checar sua identidade. Este procedimento deverá ser alterado com a finalidade de proteger os dados de acordo com a lei e ao mesmo tempo assegurar a eficiência anterior deste processo. As empresas podem se beneficiar com essa adequação, uma vez que é necessário mapear o processo como um todo da instituição, identificando problemas que, uma vez resolvidos, poderão qualificar melhor o processo, refletindo segurança ao cliente e a instituição.

\subsection{PONTOS A DESTACAR}

Como pontos comuns entre os artigos, as cartilhas e a entrevista, destacamos:

i) o avanço tecnológico, que caminha rapidamente e vem trazendo uma conexão maior ao mundo e às pessoas;

ii) o fato de que a implantação da LGPD exige a conscientização de todos os colaboradores; e

iii) a necessidade de uma gestão contínua, que implica em revisar processos e políticas constantemente para garantir que a lei está sendo efetivamente cumprida.

Em sua entrevista, o gestor menciona a figura do DPO, responsável pela proteção dos dados; nas cartilhas, essa figura é tratada como encarregado. No entanto, independentemente do termo, a LGPD preconiza essa figura.

Em particular, as cartilhas deixam clara a necessidade do consentimento dos titulares, os pacientes. Estes devem ser informados sobre os motivos de coleta daqueles dados e como eles serão tratados, assim como deverá ter acesso às políticas de privacidade da instituição. As medidas de segurança e padrões devem ser claras e as instituições devem estar adequadas a elas, com ações periódicas para monitorar a adequação e revisar processos, a fim de se evitar multas e sanções aplicadas pela ANPD pelo não cumprimento da lei.

A ANAHP informa que, quando procedimentos de segurança da informação são aplicados às informações sensíveis de saúde, combinados com atributos de 
auditoria e controles de acesso, há a prevenção de erros clínicos e a manutenção da continuidade dos serviços. De acordo com a ANAHP, no cenário de saúde, os hospitais precisam manter os prontuários físicos por, no mínimo, vinte anos para algumas patologias; em determinadas situações, este prazo pode ser ainda maior. Portanto, a digitalização desses documentos facilita seu armazenamento e gestão, ao passo que gera novas demandas para a $\mathrm{TI}$, que deve armazená-los garantindo disponibilidade e segurança. Com a evolução destes documentos para os meios digitais, a ação de manter a segurança foi elevada para níveis complexos, exigindo ambientes sofisticados, com alto custo e necessidade de atualizações constantes.

\section{CONSIDERAÇÕES FINAIS}

A cultura da organização, sem dúvida, é um dos maiores desafios para a adequação das instituições do setor de Saúde à LGPD. Dado o volume de informações que uma instituição recebe ao admitir um paciente, como controlar essas informações, para que sejam restritas a médico e paciente? Rotinas que facilitam no cuidado do paciente, como por exemplo, placas de identificação na porta do quarto ou na cabeceira e pés do leito podem trazer riscos, se não houver uma cultura baseada na LGPD.

Neste sentido, a implementação da LGPD motivará mudanças de paradigmas referentes à gestão dos dados, evidenciando a necessidade de adequações internas e da construção de uma cultura de proteção de dados na área da saúde (SÁ, 2019). Com base nas pesquisas realizadas, fica clara a necessidade de se dar segurança aos titulares dos dados e de se proteger as informações a seu respeito.

A cultura organizacional interage com os processos da instituição, onde fluxos bem definidos facilitam o aprimoramento e adequação à LGPD. Em alguns hospitais, ainda existem suportes nas portas de consultórios, utilizados para que se deixe a ficha de atendimento ou outro documento para o atendimento médico. Neste momento, vemos a necessidade do bom uso da tecnologia, onde um prontuário eletrônico evitaria que este documento fosse visto por terceiros ou mesmo extraviado. É essencial rever os processos; mapeá-los, principalmente quando nos referimos à circulação dos dados dos pacientes na instituição.

A pesquisa e a análise efetuadas mostram que todos os profissionais que trabalham em uma unidade de saúde, sem exceção, têm responsabilidade sobre os dados dos pacientes. Com a aprovação da LGPD, mais que nunca, é de suma importância que todos estejam engajados no propósito de cuidar não só da saúde, mas também, da privacidade dos pacientes. As próximas etapas a se desenvolver são o mapeamento de processos e a concretização de investimentos em tecnologia, visando adequação das instituições à LGPD.

O presente estudo revela o quanto as instituições da saúde, no segundo semestre de 2020, ainda não estão preparadas para implementar totalmente a Lei Geral de Proteção de Dados. Os desafios são vários, e vão desde a conscientização das pessoas até a realização do planejamento e efetivação dos investimentos necessários para as mudanças. O respaldo jurídico é importante, assim como o reconhecimento das ações necessárias em cada instituição. É importante observar que a LGPD é um fator potencial de motivação para impulsionar processos tecnológicos e informacionais nas instituições, proporcionando maior segurança e privacidade aos pacientes. 


\section{REFERÊNCIAS}

ANAHP - Associação Nacional de Hospitais Privados. Lei Geral de Proteção de Dados: Recomendações Anahp para os hospitais. São Paulo: ANAHP, 2019.

Disponível em: https://conteudo.anahp.com.br/cartilha-lgpd-anahp. Acesso em: 26 jun. 2021.

BRASIL. Lei $n^{\circ} 12.527$, de 18 de novembro de 2011. Regula o acesso a informações previsto no inciso XXXIII do art. $5^{\circ}$, no inciso II do $\S 3^{\circ}$ do art. 37 e no $\S 2^{\circ}$ do art. 216 da Constituição Federal; altera a Lei n 8.112, de 11 de dezembro de 1990; revoga a Lei $n^{\circ} 11.111$, de 5 de maio de 2005, e dispositivos da Lei $n^{\circ} 8.159$, de 8 de janeiro de 1991; e dá outras providências. Diário Oficial da União, Brasília, DF, p.1, 18 nov. 2011. Disponível em: http://www.planalto.gov.br/ccivil 03/ ato20112014/2011/lei//12527.htm. Acesso em: 30 jun. 2021.

BRASIL. Lei no 12.737 , de 30 de novembro de 2012. Dispõe sobre a tipificação criminal de delitos informáticos; altera o Decreto-Lei $n^{\circ} 2.848$, de 7 de dezembro de 1940 - Código Penal; e dá outras providências. Diário Oficial da União, Brasília, DF, p.1, 3 dez. 2012. Disponível em: http://www.planalto.gov.br/ccivil 03/ ato20112014/2012/lei//12737.htm. Acesso em: 30 jun. 2021.

BRASIL. Lei $n^{\circ} .12 .965$, de 23 de abril de 2014. Estabelece princípios, garantias, direitos e deveres para o uso da Internet no Brasil. Diário Oficial da União: Seção 1, Brasília, DF, ano 151, n. 77, p.1, 24 abr. 2014. Disponível em:

https://pesquisa.in.gov.br/imprensa/jsp/visualiza/index.jsp?jornal=1\&pagina=1\&data=2 4/04/2014. Acesso em: 30 jun. 2021.

DADOS do Cartão Nacional de Saúde vazam na web; ministério e PF apuram. G1, Distrito Federal, 27 mar. 2017. Disponível em: https://g1.globo.com/distritofederal/noticia/dados-do-cartao-nacional-de-saude-vazam-na-web-ministerio-e-pfapuram.ghtml. Acesso em: 30 jun. 2021.

GARCIA, Lara et al. Lei Geral de Proteção de Dados Pessoais (LGPD): Guia de Implantação. São Paulo: Editora Blucher; Fundação Vanzolini, 2020.

GREGORI, Maria S. Os Impactos da Lei Geral de Proteção de Dados Pessoais na Saúde Suplementar. Revista de Direito do Consumidor, São Paulo, v. 127, p.171-196, jan./fev. 2020. Disponível em: https://revistadedireitodoconsumidor.emnuvens.com.br/ rdc/article/view/1268/1189. Acesso em: 29 jun. 2021.

INFORMATIVO de Legislação Federal. Gen Jurídico, São Paulo, 11 jul. 2018. Disponível em: http://genjuridico.com.br/2018/07/11/informativo-de-legislacao-federal11-07-2018/. Acesso em: 24 jun. 2021.

JINKINGS, D. Governo vai debater criação de marco legal para proteção de dados pessoais no Brasil. Rede Brasil Atual, São Paulo, 1 dez. 2010. Disponível em: https://www.redebrasilatual.com.br/cidadania/2010/12/governo-vai-debater-criacao-demarco-legal-para-protecao-de-dados-pessoais-no-brasil/. Acesso em: 16 ago. 2020. 
LUIZ, Gabriel; RODRIGUES, Matheus. MP do DF apura se Cambridge Analytica usou dados de brasileiros no Facebook. G1, Distrito Federal, 21 mar. 2018. Disponível em: https://g1.globo.com/df/distrito-federal/noticia/mp-do-df-apura-se-cambridge-analyticausou-dados-de-brasileiros-no-facebook.ghtml. Acesso em: 30 jun. 2021.

NASCIMENTO, Luciano. Governo publica MP que cria órgão para proteção de dados. AgênciaBrasil, Brasília, 28 dez. 2018. Disponível em:

https://agenciabrasil.ebc.com.br/geral/noticia/2018-12/governo-publica-mp-quecria-orgao-para-protecao-de-dados. Acesso em 29 jun. 2021.

PIURCOSKY, Fabricio P. et al. A lei geral de proteção de dados pessoais em empresas brasileiras: uma análise de múltiplos casos. Suma de Negócios, Bogotá, CO, v. 10, n. 23, p. 89-99, jul./dez. 2019. Disponível em:

http://www.scielo.org.co/pdf/sdn/v10n23/2215-910X-sdn-10-23-89.pdf. Acesso em: 29 jun. 2021.

SÁ, Marcelo D. Análise do impacto da Nova Lei de Proteção de Dados Pessoais nas aplicações de Internet das coisas: Aplicações mobile do governo. 2019.

Monografia (especialização em Informática) - Universidade Federal de Minas Gerais, Departamento de Ciência da Computação, Belo Horizonte, 2019.Disponível em: https://repositorio.ufmg.br/bitstream/1843/32040/1/MarceloDiasDeSa.pdf. Acesso em: 29 jun. 2021.

SARLET, Gabrielle Bezerra S.; MOLINARO, Carlos A. Questões Tecnológicas, Éticas e Normativas da Proteção de Dados Pessoais na Área da Saúde em um Contexto de Big Data. Direitos Fundamentais e Justiça, Belo Horizonte, v. 13, n. 41, p. 183-212, jul./dez. 2019. Disponível em: http://dfj.emnuvens.com.br/dfj/article/view/811/964. Acesso em: 28 jun. 2021.

SCHRAMM, Julie Katlyn A. A era biotecnológica: apontamento sobre os dados genéticos na LGPD (Lei Geral de Proteção de Dados). In: EVENTO DE INICIAÇÃO CIENTÍFICA - EVINCI, 14., 2019, Curitiba. Anais eletrônico [...]. Curitiba: UniBrasil, 2019. v.5, n.1. Caderno de resumos. Disponível em:

https://portaldeperiodicos.unibrasil.com.br/index.php/anaisevinci/article/view/5098/386 5. Acesso em: 29 jun. 2021.

SOUZA, Allan R. et al. Marcos legais nacionais em face da abertura de dados para pesquisa em saúde: dados pessoais, sensíveis ou sigilosos e propriedade intelectual. Rio de Janeiro: Fiocruz, 2018. 123 p. Disponível em: https://www.arca.fiocruz.br/handle/icict/28838. Acesso em: 29 jun. 2021.

UNIÃO EUROPEIA. Parlamento Europeu e Conselho. Regulamento Geral sobre a Proteção de Dados n. 2016/679. Jornal Oficial da União Europeia, Bruxelas, p. L 119/1-L 119/88, 2018. Disponível em: https://eur-lex.europa.eu/legalcontent/\%20PT/TXT/PDF/?uri=CELEX:32016R0679\&from=en. Acesso em: 26 jun. 2021.

WARREN, Samuel D.; BRANDEIS, Louis D. The right to privacy. Harvard Law Review, v. 4, n. 5, p.193-220, Dec.1890. Disponível em: http://groups.csail.mit.edu/mac/classes/6.805/articles/privacy/Privacy brand warr2. 
html. Acesso em: 30 jun. 2021. 